\title{
Impella 5.0 repositioning across the aortic valve without a guidewire using rapid ventricular pacing: A case report
}

\author{
Paolo Masiello ${ }^{1}$, Francesco Frunzo ${ }^{1}$, Marco Padula ${ }^{1}$, Generoso Mastrogiovanni ${ }^{1}$, Donato \\ Triggiani $^{1}$, Alessandro Laudani ${ }^{1}$, Rocco Leone ${ }^{1}$, and Severino Iesu ${ }^{1}$ \\ ${ }^{1}$ University Hospital 'San Giovanni di Dio e Ruggi d'Aragona'
}

April 29, 2020

\begin{abstract}
Background Displacement of Impella 5.0 secondary to patient movement or transportation is a well known complication. Typically, repositioning of an Impella across the aortic valve is attempted over a guidewire. We present the first case, to our knowledge, of repositioning a dislodged Impella 5.0 without a guidewire under transesophageal echocardiography (TEE) guidance, by inducing rapid ventricular pacing to cross the aortic valve. Case presentation: A 70-year-old man with low left ventricular ejection fraction underwent off-pump coronary artery bypass grafting (OPCABG). On 2nd postoperative day a low cardiac output state developed with increasing lactate levels and consequently the patient was taken to the cardiac catheterization laboratory for insertion of an Impella 5.0. Suddenly the Impella system failed with a rapid hemodynamic deterioration and it was successfully bedside repositioned inducing rapid ventricular pacing. Conclusions: In case of accidental Impella dislodgement and fast deterioration of patient's hemodynamic status, rapid pacing may be an option to "open" the aortic valve thus aiding quick replacement of Impella 5.0 through the aortic valve into the left ventricle under TEE guidance.
\end{abstract}

\section{BACKGROUND}

Percutaneous mechanical circulatory support with Impella 5.0 (Abiomed, Danvers, MA) is increasingly used in the treatment of cardiogenic shock due to its effectiveness, userfriendlyness and the relatively easy management in the ICU. ${ }^{1,2}$ However, the displacement of Impella devices secondary to patient movement or transportation is a known complication. ${ }^{3,4}$

Dislodgement of Impella can lead to migration of the device deep into the ventricular cavity or out into the aortic root with subsequent failure of ventricular unloading. Device repositioning is resource-intense, requiring a hybrid operating room or catheterization lab and a multidisciplinary team with close cooperation between trained ICU physicians, nursing staff and cardiologist. ${ }^{5}$ Also, improper Impella positioning can result in hemolysis related to increased shear stress and mechanical irritation of adjacent cardiac structures such as papillary muscles.

We report a case of successful bedside repositioning of an Impella 5.0 without guidewire under TEE guidance, facilitated by rapid ventricular pacing to cross the aortic valve. To the best of our knowledge this is the first time that this technique is reported.

\section{CASE PRESENTATION}

A 70-year-old man was re-hospitalized one month after initially suffering an anterior non-ST-elevation myocardial infarction (NSTEMI) with pulmonary edema. Coronary angiography revealed severe left main and triple vessel disease, and echocardiography showed severe left ventricular dysfunction with ejection fraction (EF) of $32 \%$. 
Therefore, the patient underwent coronary artery revascularization using off-pump coronary artery bypass grafting (OPCABG). General anesthesia was induced using propofol-remifentanil target-controlled infusion and off-pump three-vessel CABG surgery was performed. The patient was hemodynamically unstable during the procedure and received inotropes and vasopressors (dobutamine $5 \mathrm{mcg} / \mathrm{kg} / \mathrm{min}$, norepinephrine and adrenaline $0.1 \mathrm{mcg} / \mathrm{kg} / \mathrm{min}$ ). Given the improving clinical conditions with stable EF, pharmacological therapy was gradually reduced in the postoperative period.

On postoperative day (POD) 2, the patient developed atrial fibrillation with high ventricular rate, not responsive to any treatment, resulting in a low cardiac output state with increasing lactate levels. The EF rapidly worsened, and the patient was taken to the cardiac catheterization laboratory for insertion of an Impella 5.0. The Impella was positioned through a 10-mm Dacron graft anastomosed end-to-side to the right common femoral artery. The position of device was confirmed by fluoroscopy. The patient was then transferred in ICU. Following transfer, the Impella system failed with a rapid hemodynamic deterioration of the patient. The cardiology team performed a bedside TEE to check the device position. TEE showed migration of the Impella distal tip into the ascending aorta (Fig. 1).

Repositioning of the device was attempted at bedside under TEE guidance. However, the biggest challenge was to pass the device through the aortic valve without a guidewire. To overcome this challenge, we used a procedural step usually employed during transcatheter valvuloplasty. With the epicardial leads left in place after the operation, rapid ventricular pacing to $200 \mathrm{bpm}$ was induced to keep the aortic valve almost motionless and in a "systolic" position. The Impella was then directed through the aortic valve and advanced into the left ventricle. The procedure was easy and fast, the position of the Impella was rapidly optimized and support resumed with maximal flow (Fig. 2). Impella 5.0 was weaned after 8 days of optimal support without any other complications. TEE performed after removal showed no any aortic valve damage.

\section{DISCUSSION AND CONCLUSIONS}

The increased adoption of percutaneous ventricular assist devices in the current era may be supported with a series of "tips and tricks" based on clinical experience. While the use of Impella devices is increasing, complications such as hemolysis and ventricular tachycardia are being reported. An important requirement for prolonged optimal hemodynamic support with Impella is therefore optimal ICU management and positioning.

The Impella 5.0 is a microaxial pump that works based on the principle of an Archimedes' screw. It is a transvalvular pump positioned across the aortic valve that propels blood from the left ventricle into the ascending aorta. The pigtail located at the distal tip of the cannula aids in stabilizing the catheter in the correct position. Impella 5.0 is inserted via the femoral or axillary artery surgically using a side graft anastomosed to the artery. In this case femoral artery was used due to technical problems in approaching the axillary artery (obesity and diffuse supra-aortic vessels atherosclerosis). The Impella is placed in the catheterization laboratory under fluoroscopy or in the hybrid operating room, if available. Evaluation of Impella position can be performed using bedside TEE, and we report safe repositioning given the adequate visualization of Impella in relation to cardiac structure such as mitral sub-valvular apparatus or the aortic valve. ${ }^{6}$ Typically, the patient is brought back to the catheterization laboratory or a hybrid operating room, and the Impella is advanced over a guidewire to cross the aortic valve.

In the present case, the guidewire was no longer in place, a hybrid operating room was not available, and the patient's clinical condition deteriorated rapidly. The on duty team identified the challenge of crossing the aortic valve and decided to use the technique of rapid pacing to keep the aortic valve almost "motionless". Following this approach, the pigtail and cannula were introduced in the left ventricle without a problem or injury to the aortic valve, as confirmed by TEE (Fig. 2). The final positioning was confirmed on TEE with the inlet of the device located in the left ventricle about $3.5 \mathrm{~cm}$ below the aortic valve.

We acknowledge that this trick of repositioning a dislodged Impella 5.0 cannot be routinely employed in the clinical situation, given the risk of rapid ventricular pacing and LV wall or aortic valve damage. This is the 
first described case at our institution where an Impella 5.0 was successfully repositioned across the aortic valve without a guidewire at bedside under TEE guidance, and to our knowledge the first reported case in the literature using this technique.

In case of accidental Impella dislodgement and fast deterioration of patient's hemodynamic status, rapid pacing may be an option to "open" the aortic valve safely, thus aiding in placement of Impella 5.0 through the aortic valve into the left ventricle. In case of epicardial leads absence a transvenous temporary pacing lead can be positioned without fluoroscopy but the "time consuming" must be evaluated.

Author contributions: Dr. Masiello, Dr. Frunzo, Dr. Padula and Dr. Triggiani developed the concept and designed the study. Drafting the article was made by Dr. Masiello and Dr. Mastrogiovanni, Critical revision was made mainly by Dr. Iesu. All other authors contributed to data analysis collection, statistics, critical revision and approval of the article.

\section{References}

1. Batsides G, Massaro J, Cheung A, et al. Outcomes of Impella 5.0 in Cardiogenic Shock: A Systematic Review and Meta-analysis.Innovations (Phila) 2018; 13: 254-60. DOI: 10.1097/IMI.0000000000000535; PMID: 30142110.

2. Griffith BP, Anderson MB, Samuels LE, et al. The RECOVER I: a multicenter prospective study of Impella 5.0/LD for postcardiotomy circulatory support. J Thorac Cardiovasc Surg 2013;145:548-54. DOI: 10.1016/j.jtcvs.2012.01.067; PMID: 22405676.

3. Mastroianni C, Bouabdallaoui N, Leprince P, Lebreton G. Short-term mechanical circulatory support with the Impella 5.0 device for cardiogenic shock at La Pitié-Salpêtrière.Eur Heart J Acute Cardiovasc Care 2017;6:87-92. DOI: 10.1177/2048872616633877; PMID: 26873912.

4. Kaluski E, Khan SU, Sattur S, Sporn D, Singh M. Impella CP Dislodgment, Swap, or Removal: Current Practices.J Invasive Cardiol 2019;31(1):36-40. PMID: 30611124.

5. Burzotta F, Trani C, Doshi SN, et al. Impella ventricular support in clinical practice: Collaborative viewpoint from a European expert user group. Int J Cardiol 2015; 201:

684-91. DOI: 10.1016/j.ijcard.2015.07.065; PMID: 26363632.

Crowley J, Cronin B, Essandoh M, et al. Transesophageal Echocardiography for Impella Placement and Management. J Cardiothorac Vasc Anesth 2019;33:2663-68. DOI: 10.1053/j.jvca.2019.01.048; PMID: 30770179.

Figures legend

Figure 1: displacement of Impella's tip

Figure 2: Impella repositioning 

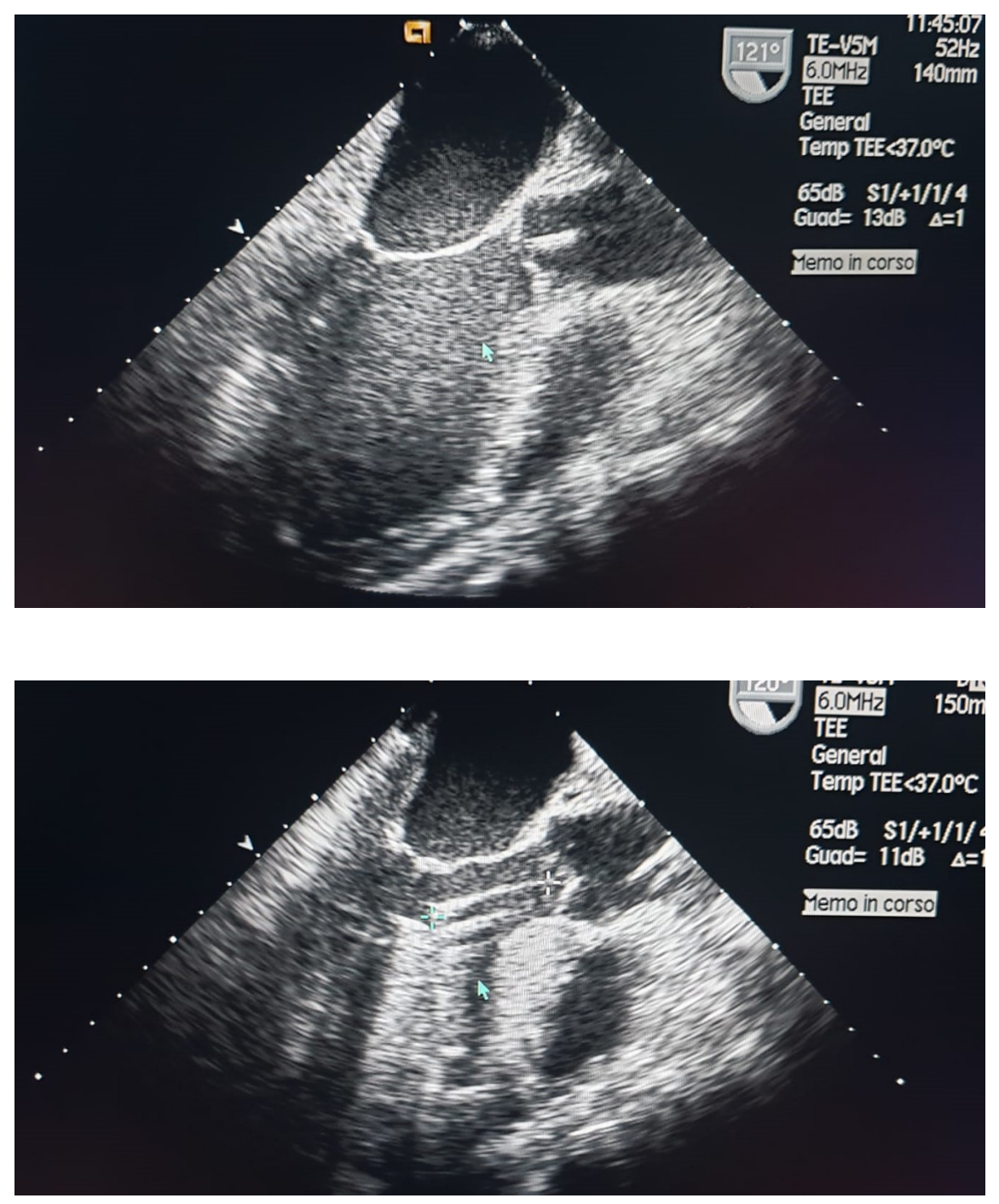